\title{
Design of an Occupancy Monitoring Unit: A Thermal Imaging Based People Counting Solution for Socio- Technical Energy Saving Systems in Hospitals
}

\author{
Ahmad Taha, Jan Krabicka \\ School of Engineering \\ University of Greenwich \\ Kent, United Kingdom \\ A.M.Taha, \\ J.Krabicka\}@Greenwich.ac.uk
}

\author{
Ruiheng $\mathrm{Wu}$ \\ School of Engineering and the \\ Built Environment \\ Birmingham City University \\ Birmingham, United Kingdom \\ Ruiheng.Wu@bcu.ac.uk
}

\author{
Peter Kyberd \\ School of Energy and \\ Electronic Engineering \\ University of Portsmouth \\ Portsmouth, United Kingdom \\ Peter.Kyberd@port.ac.uk
}

\author{
Neil Adams \\ Clinical Engineering \\ Medway NHS FT \\ Kent, United Kingdom \\ Neil.adams5@nhs.net
}

\begin{abstract}
Aside from their physical exterior/interior design, the energy demands of industrial buildings are strongly related to how they are used. It follows that the behaviour of the occupants contributes and is related to the energy consumption of a building. In a hospital, this could mean equipment usage, heating, water and so on. This implies that energy consumption in a specific area may be measured as a function of occupancy, making occupancy monitoring an important part of an organisation's energy management plan. This paper presents the design, implementation and testing of an Occupancy Monitoring Unit (OMU), based on thermal imaging technology, to provide occupancy data on individual selected wards/areas of Medway NHS Foundation Trust (MWNFT). The paper also presents successful tests performed to assess the functionality of the OMU in counting people and identifying the direction of motion. The results show the potential for the OMU to count individual people as well as groups.
\end{abstract}

Keywords- Occupancy Monitoring; Thermal Imaging; Embedded Systems; Occpuants' Behaviour

\section{INTRODUCTION}

The energy activity in a building relies mostly on the presence of occupants within it [1]. However, a clear relationship between physical occupancy and energy consumption is yet to be established. Take, for example, leaving a PC monitor 'on' after working hours. Although the action was taken whilst physically present, the energy impact will be sustained after the individual has left.

This paper presents a proposed system, the Occupancy Monitoring Unit (OMU), that provides data on occupancy by counting the people entering and exiting a confined space in a building. Whilst the aim is to design it for a hospital, as part of a bigger research project that studies the impact of technology on pro-environmental behaviour [2], to count people in a ward/department throughout the day; the system can be used in other types of buildings such as universities, commercial buildings and in public transportation. The design, implementation and testing of a prototype OMU to provide data on occupancy for analysis and management of energy consumption is presented. The remainder of the paper is structured as follows:
Sections II and III review existing literature concerning the relationship between occupancy and energy consumption (section II) and people counting technologies (section III). Section IV presents the design of the OMU and sections V and VI detail its implementation and testing. Section VII concludes the paper and provides directions for future work.

\section{BUILDING OCCUPANCY AND ENERGY CONSUMPTION}

Several studies discuss the introduction of new energy measures without accounting for occupancy, which can skew forecasted energy consumption to up to $300 \%$ [3]. This indicates a strong relation between occupancy and energy consumption and highlights the importance of monitoring rates of occupancy.

The Rowner research project investigated the design and delivery of two blocks of flats, as part of the Building Performance Evaluation (BPE) programme. The project was named after the village that it was piloted in. Findings show a considerable difference in energy consumption between the two block of flats that were involved in the study [4]. The authors linked this big difference to occupancy levels and occupants' thermal preferences. Another study, [5], monitored the heat energy usage in three identical dwellings located in the Netherlands. Large variations were found that were reasoned back to occupancy levels and occupants' behaviour.

Results from, [6], have shown that there is a strong correlation between occupancy and the use of electrical appliances, mostly personal computers (PCs). The anticipation is for consumption to decrease outside of working hours or during lunch times. The authors noted that the energy consumption did not change significantly in time periods like lunch, where majority of the participants were not at their desks, which flagged a behavioural issue. Although computers and their monitors do save electricity when in idle mode, their consumption drops to about a third and hence would be better off shutdown if not in use, for example over lunch time or when leaving for the evening [7]. Staff members in various organisations tend to leave their PCs and their monitors 'on' to easily resume work after lunch or even the following day.

With low power technology now part of many of the newly produced electronic based devices, the tendency to leave 
equipment to go into sleep/idle/power save mode without worrying about switching them 'off' has increased, hence creating negative behavioural patterns. The previously discussed examples from the literature shows the importance of considering occupancy levels and therefore the positive impact an OMU can have as part of an energy management plan.

\section{OVERVIEW OF PEOPle COUNTING TeChNOlogies}

Several methods have been adopted to implement people counting systems using sensor technology. However, the environment in which the sensor is to operate plays an important role in deciding on what technology to use. In a hospital environment, it is important to consider data protection, that is anonymity of the people, as well as accurate detection of people whether they are as individuals or in groups. Prior research work has proposed the use of various technologies such as Radio Frequency Identification (RFID) [6], [8], Wi-Fi [9] and sensor networks [10], [11], [12], [13] to monitor occupancy.

One of the proposed technique was to count the number of available Wi-Fi connections [9]. This would indicate the level of occupancy in the area covered by the wireless network. The aim was to identify whether the occupancy has a high impact on the energy consumption in Massachusetts Institute of Technology (MIT).

RFID is a commonly used technology in various sectors of the society as it increases productivity and convenience. It is an obvious people counting solution due to its real-time feature, accuracy and robustness of the technology, which has been around since the 1970s [14]. Two studies [6], [8] investigated the use of RFID to monitor human occupancy against energy efficiency and efficient building lighting control respectively. The first,[6], investigated the effect of combining occupancy monitoring using RFID with Passive Infrared (PIR) sensors on building lighting control and showed good results of accuracy.

In reference [6] the author reviews different measurement technologies such as the use of motion sensors, CO2 levels, acoustics, Radio Frequency (RF) signals, and so on, while classifying them according to their function and the method they use. The review highlighted the advantages and disadvantages of each technology and whether they can provide accurate occupancy information alone or combined. The authors settled for the use of RFID in their study due to its intermediate cost and accuracy compared to other solutions.

Table I presents a brief comparison between four sensor technologies (Wi-Fi, RFID, Beam Sensors, and Thermal Imaging) highlighting the key points by which the sensor selection was made. Although several other technologies were discussed, the table focuses on the common technologies that are easily available in the market.

TABLE I. COMPARISON BETWEEN VARIOUS SENSOR TECHNOLOGIES FOR OCCUPANCY MONITORING

\begin{tabular}{|c|l|}
\hline People Counting Technology & \multicolumn{2}{|c|}{ Operation and limitations } \\
\hline Wi-Fi & $\bullet \begin{array}{l}\text { Uses the number of Wi-Fi } \\
\text { connections to an access point } \\
{[9] .}\end{array}$ \\
\hline
\end{tabular}

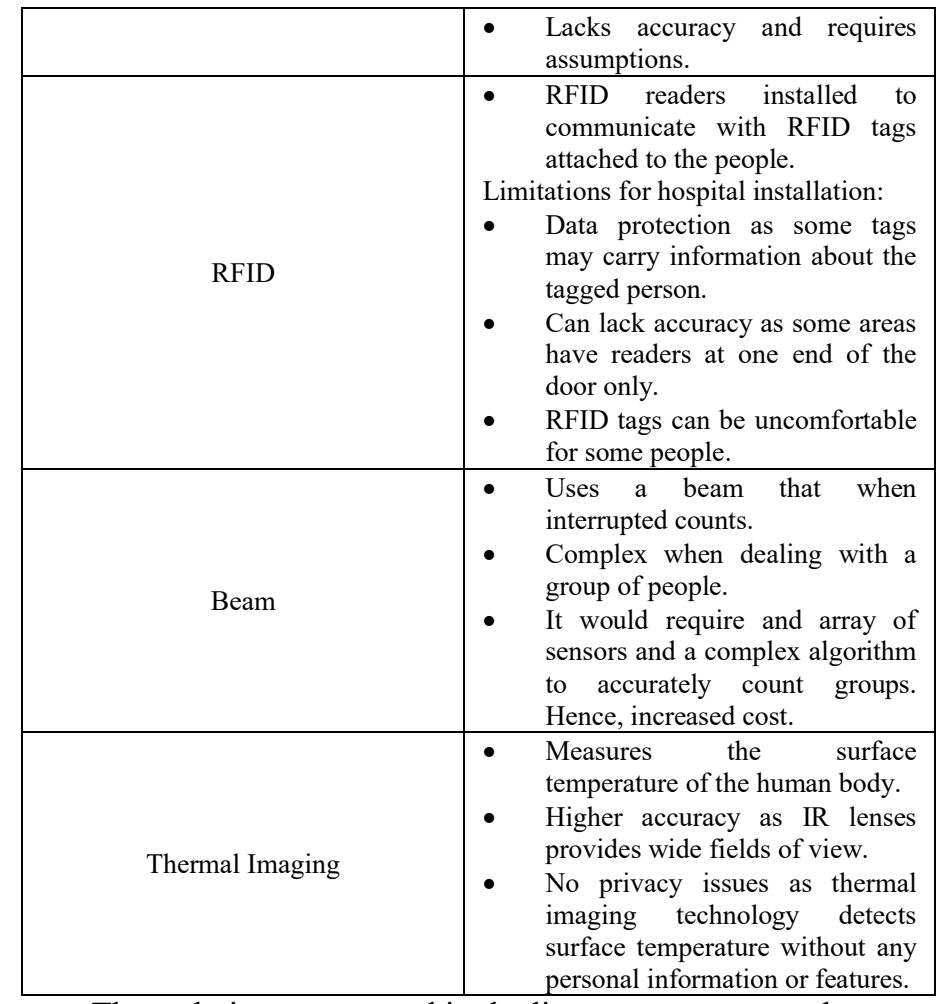

The techniques proposed in the literature to measure human occupancy show good results in relation to the aims set by the researchers who conducted the studies. The proposed techniques involved the use of Wi-Fi, RFID, PIR and others. Some techniques were suitable for the aim but would not fit a hospital environment, while others lack accuracy, as presented within the literature. For instance, with Wi-Fi, the assumption that each person present on the MIT campus has only one WiFi enabled device is crucial to ensure high accuracy and this cannot be guaranteed. Although wireless technology currently represents a handy and cheap solution in all sectors of the society, using them for people counting does not represent the optimum solution as the accuracy of the readings may vary significantly. On the other hand, using RFID in a hospital environment would make it difficult to tag people with RFID tags.

Accordingly, and with reference to Table I, Omron's Thermal Imaging Sensor (TIS), D6T-44L-06 [15], was selected for the design of the OMU. It was found to be the more realistic technology to go with compared to the rest in terms of complexity, principle of operation and for proofing the concept of the OMU as part of the socio-technical behavioural change system for MWNFT.

\section{OCCUPANCY MONITORING UNIT DESIGN}

This section of the paper presents the hardware and software designs of the OMU. An overview of the TIS is presented at first as its working principle is crucial for the design of the OMU.

\section{A. Overview of the Thermal Imaging Sensor D6T-44L}

Omron's TIS is composed of an array of 16 thermal sensing elements in the form of a $4 \times 4$ matrix as seen in Fig. 1. The 
temperature recorded by each element increases when a human is within its zone and decrease when the human leaves. The motion can be identified by recording the temperature of the pixels and analyse the changes in temperature of the adjacent pixels. Hence, indicating a motion in a specific direction.

The TIS incorporates a Microelectromechanical Systems (MEMS) thermopile that measures the surface temperature of an object by detecting the intensity of the infrared radiation. The sensor's Field of View (FoV) has a horizontal viewing angle of $44.2^{\circ}$ and a vertical viewing angle of $45.7^{\circ}$. The sensor sends a 35-byte packet holding the data for the 16 pixels (two bytes for each pixel) along with the reference temperature and the CRC check byte. The temperature data is a 16-bit width, signed and 10 times the value of the actual temperature. The packet is sent serially through an onboard Two-Wire Interface (TWI) [16]. Each pixel represents the noncontact temperature measured by the sensor, the shorter the distance is between the object and the sensor, the more accurate it is. However, it is capable of detecting temperature of objects as far as $10 \mathrm{~m}$ from the sensor's lens [15], [17].

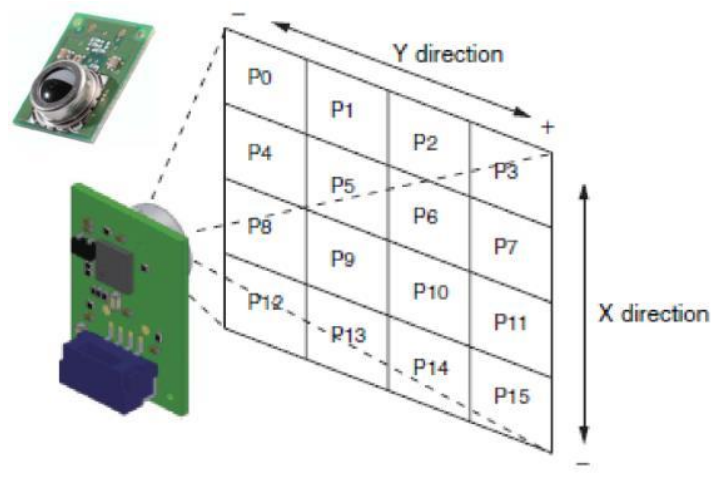

Figure 1. Omron's D6T-44L TIS pixel distribution [15]

\section{B. Hardware Design}

A simple block diagram, Fig. 2, was developed to indicate the main components that form the OMU.

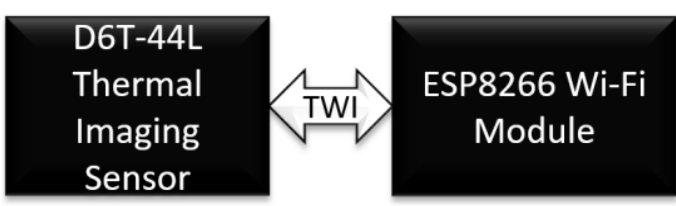

Figure 2. OMU block diagram

Pixel temperatures captured by the TIS are sent over the TWI to the onboard Microcontroller Unit (MCU) of the ESP8266 Wi-Fi module. ESP8266 Wi-Fi module is commonly used for Internet of Things based applications [18], [19], due to its low cost and low power features. The Wi-Fi module has a range of 50-100 meters and unlike other long-range wireless communication technologies, it can be used in a hospital. The ESP8266 module could be programmed using the Arduino Integrated Development Environment (IDE) and is compatible with several interfacing libraries.

\section{Software Design - Human Detection and Direction Algorithm}

The software for the OMU was built on two stages. first stage was to make sure that both sides, see Fig. 2, communicated together and this was a crucial part of the software design stage to ensure the functionality of the algorithm for the OMU. Fig. 3 shows some data read and processed from the sensor with (right-hand side of the figure), and without (left-hand side of the figure) a test subject (waving a hand) within the its FoV.

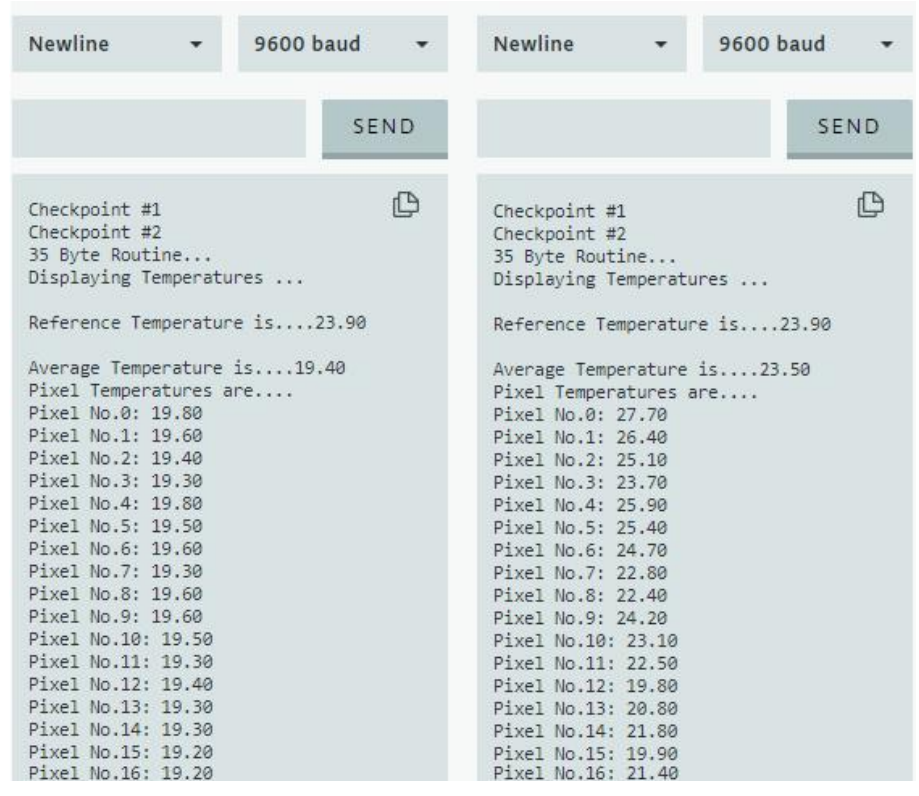

Figure 3. Two Sets of temperature readings showing the sensor's response to waving a hand across its FoV

Once powered, the D6T-44L TIS continuously captures a thermal image of the area covered by its FoV. The TWI is a Master/Slave synchronous serial interface where the MCU is the master and the D6t-44L TIS is the slave. To establish the communication link that results in pixel temperatures communicated to the MCU, see Fig. 3, the MCU sends a series of commands over the TWI data line (SDA) to request the data from the sensor.

The Human Detection and Direction (HDD) algorithm, designed to count the number of humans, was built around the operating concept of the sensor. Fig. 4 is a flowchart of the HDD algorithm designed to count the number of people entering or exiting through a door at any one time. The algorithm uses the orientation of the sensor's $4 \times 4$ matrix of pixels, see Fig.1, and the sequence in which the pixels are triggered to identify direction of motion which is crucial to identify whether a person is entering or exiting. The number of pixels triggered in a single row in one reading are used to log whether a single person or two persons are entering/exiting at the same time. Some assumptions were made during the design phase for proof of concept and rapid prototyping purposes:

- A maximum of two people will enter/exit at the same time. Apart from easing the design, the entry/exit doors of the 
pilot wards/departments allow for a single person entry/exit but can allow two people if opened widely.

- A scenario where a person enters and another one exits at the same time was not considered in the design as it was unlikely to happen.

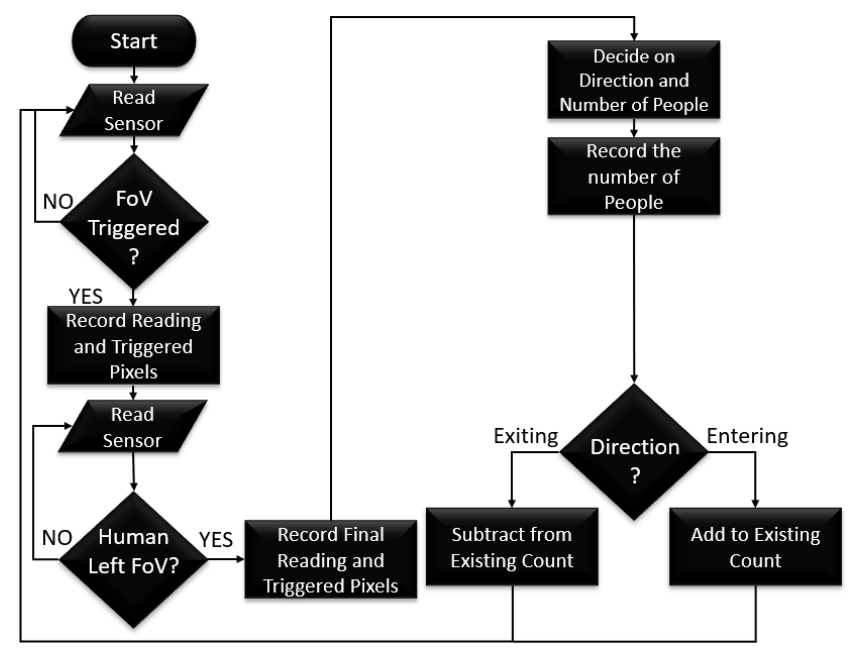

Figure 4. The OMU's HDD algorithm flowchart

\section{IMPLEMENTATION}

Having introduced the design, this section presents the hardware and software implementation of the OMU.

\section{A. Hardware Implementation}

A prototype was built for the OMU on a breadboard, as seen in Fig. 5, which was based on the connections shown in Fig. 6. A breadboard was used instead of a Printed Circuit Board (PCB) because the electronic modules, used, all sat on pin header connectors, so a PCB would have been a breakout board for the modules. Hence, for rapid prototyping purposes, a breadboard was used.

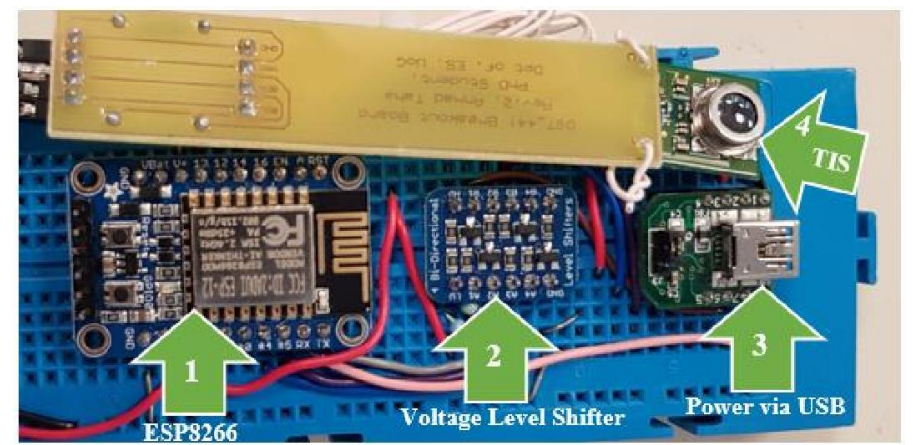

Figure 5. OMU working prototype

The module labelled " 3 ", in Fig. 5, is a UB232R Universal Serial Bus (USB) converter, which was used to power the board from a laptop or a USB powering source. The UB232R module also acted as a communication channel between the OMU's Universal Asynchronous Receiver-Transmitter (UART) and the serial monitor on a PC or a laptop, as demonstrated in Fig. 3 and similar to [20]. The module labelled
"2", in Fig. 5, is a voltage level shifter that converts voltage levels from $5 \mathrm{~V}$, that is, Transistor Transistor Logic (TTL) to, $3.3 \mathrm{~V}$, that is, Low Voltage TTL (LVTTL). The level shifter was used to translate the 5V, SDA and clock (SCL) lines of the D6T-44L TIS, to the ESP8266 3.3V level.

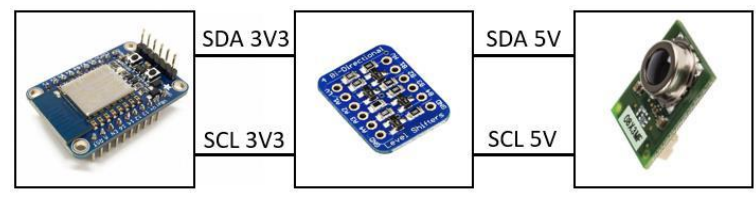

Figure 6. Module connections of the OMU

\section{B. Software Implementation}

The algorithm shown in Fig. 4 was coded on an Arduino IDE and programmed to the ESP8266's on board MCU following the hardware implementation phase.

The algorithm relies on the change in the sensed temperature within the FoV to $\log$ the presence of a human. To ensure accurate detection of a human, a "FoV Trigger" test was made were the TIS was attached to the ceiling of a room, see Fig. 7, and using the height of the sensor and the FoV angles, the TIS's $4 \times 4$ matrix was marked on the floor. A human Test Subject (TS) was used to record the temperature of each pixel when the TS was standing within its area. The recorded pixel temperature readings were subtracted from a FoV thermal image without the TS and data were plotted, see Fig. 8. The plotted data were for 9 out of the 16 pixels as they were the most likely to be impacted with the TIS's orientation seen in Fig. 7. The recorded temperatures showed a difference of 5 degrees or higher, in the TS pixel location, as indicated in Fig. 8. Accordingly, the "FoV Triggered" and "Human Left FoV" blocks in the flowchart of Fig. 4, were implemented.

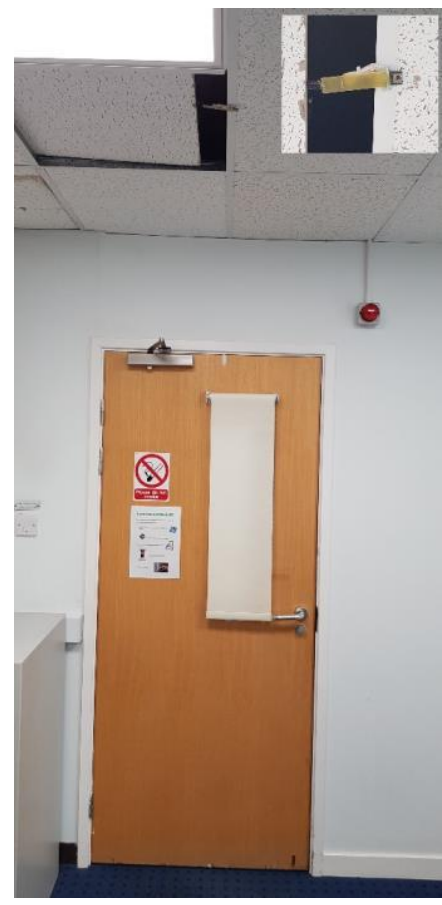

Figure 7. D6T-44L TIS attached to the ceiling for testing 


\section{TEMPERATURE DIFFERENCE WITH AND WITHOUT A HUMAN TEST SUBJECT}

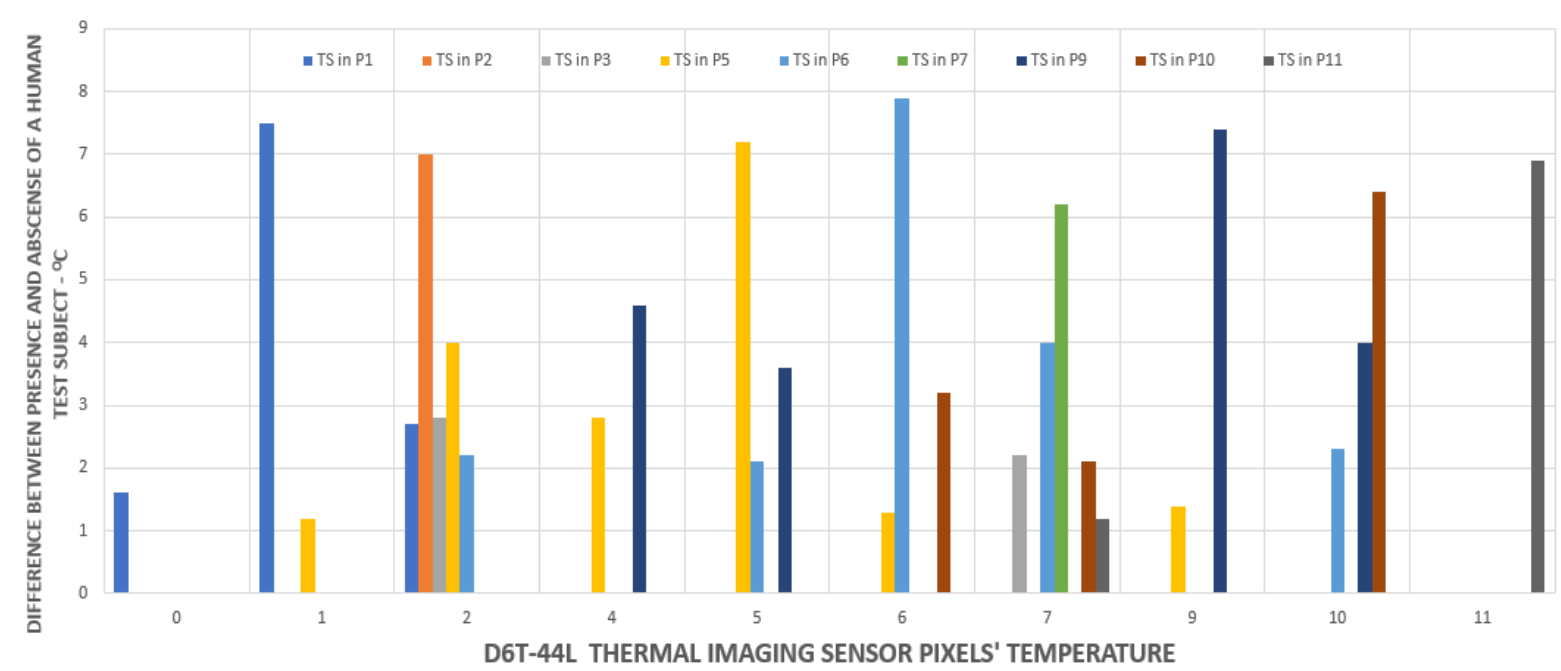

Figure 8. Difference in pixel temperatures between a thermal image with a human TS and an empty thermal Image

\section{TESTING AND PROOF OF CONCEPT}

Several tests were performed to assess the functionality of the OMU. The performed tests were set to evaluate the OMU functionality and to demonstrate its working principle. The first test performed involved a human TS walking within the TIS's FoV as represented by Fig. 9. A Graphical User Interface (GUI) was implemented to visualise the sensor's FoV, in realtime, in the form of an onscreen $4 \times 4$ Matrix of pixel temperatures, Fig. 10.
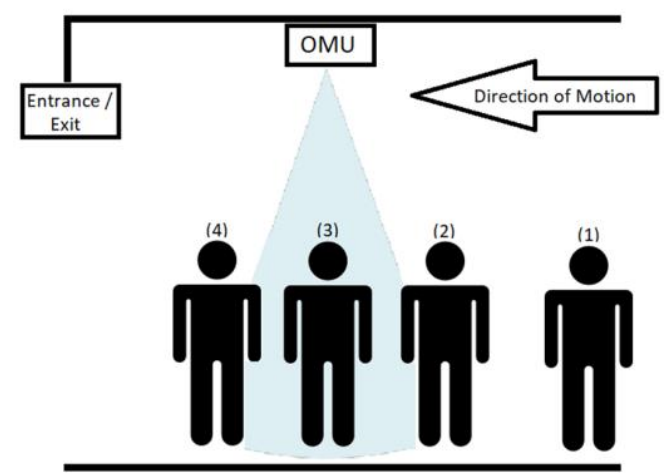

Figure 9. Demonstration of the motion of a human TS walking through the sensor's FoV in the performed test

The labels (1), (2), (3) and (4) on the captured thermal images in Fig. 10 reflect those taken by the sensor when the human TS was in different locations within its FoV, as seen in Fig. 9. This test was performed using the same human TS that was used to generate the data presented in Fig. 8.

The human TS, along the walking path, occupied eight pixels, Fig. 10 (2), (3) and (4), from the matrix. Three to four pixels were occupied at any one time, Fig. 10 (2) \& (4), however also depending on the person's size and motion, that

is, in a straight line or not. This shows the potential of the system to count a group of people, at the same time. Nevertheless, as mentioned earlier, the OMU presented in this paper was designed and tested to count a single person or multiple consecutive persons either entering or exiting or alternating between both; a common scenario in MWNFT due to the limited available space.

(2)
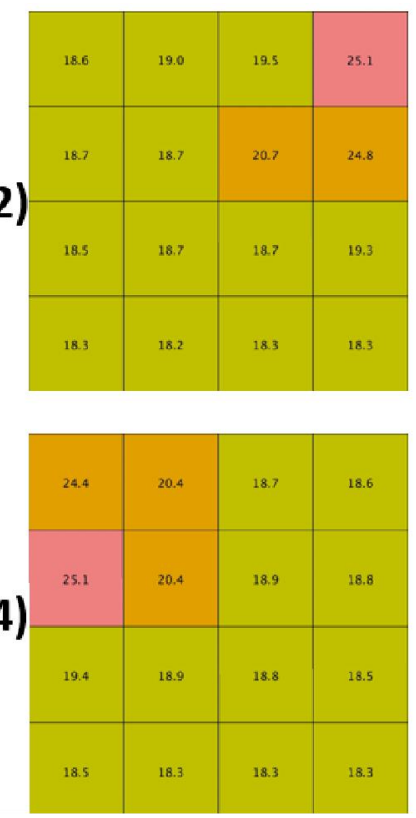

Figure 10. A GUI Showing four thermal captures of a human TS passing through the sensor's FoV

Further tests, using three human TSs, were performed to test the HDD algorithm. Table II shows the performed tests. 
